\title{
Comparison of Carotid Plaque Ulcer Detection Using Contrast- Enhanced and Time-of-Flight MRA Techniques
}

\author{
M. Etesami, Y. Hoi, D.A. Steinman, S.K. Gujar, A.E. Nidecker, B.C. Astor, A. Portanova, Y. Qiao, W.M.A. Abdalla, and B.A. Wasserman
}

- $D$

\begin{abstract}
BACKGROUND AND PURPOSE: Ulceration in carotid plaque is a risk indicator for ischemic stroke. Our aim was to compare plaque ulcer detection by standard TOF and CE-MRA techniques and to identify factors that influence its detection.
\end{abstract}

MATERIALS AND METHODS: Carotid MR imaging scans were acquired on 2066 participants in the ARIC study. We studied the 600 thickest plaques. TOF-MRA, CE-MRA, and black-blood MR images were analyzed together to define ulcer presence (plaque surface niche $\geq 2 \mathrm{~mm}$ in depth). Sixty ulcerated arteries were detected. These arteries were randomly assigned, along with 40 nonulcerated plaques from the remaining 540, for evaluation of ulcer presence by 2 neuroradiologists. Associations between ulcer detection and ulcer characteristics, including orientation, location, and size, were determined and explored by CFD modeling.

RESULTS: One CE-MRA and 3 TOF-MRAs were noninterpretable and excluded. Of 71 ulcers in 56 arteries, readers detected an average of $39(55 \%)$ on both TOF-MRA and CE-MRA, 26.5 (37.5\%) only on CE-MRA, and 1 (1.5\%) only on TOF-MRA, missing 4.5 (6\%) ulcers by both methods. Ulcer detection by TOF-MRA was associated with its orientation (distally pointing versus perpendicular: $\mathrm{OR}=5.57[95 \% \mathrm{Cl}$, 1.08-28.65]; proximally pointing versus perpendicular: $\mathrm{OR}=0.21$ [95\% $\mathrm{Cl}, 0.14-0.29]$ ); location relative to point of maximum stenosis (distal versus isolevel: $\mathrm{OR}=5.17[95 \% \mathrm{Cl}, 2.10-12.70])$; and neck-to-depth ratio $(\mathrm{OR}=1.96[95 \% \mathrm{Cl}, 1.11-3.45])$ after controlling for stenosis and ulcer volume.

CONCLUSIONS: CE-MRA detects more ulcers than TOF-MRA in carotid plaques. Missed ulcers on TOF-MRA are influenced by ulcer orientation, location relative to point of maximum stenosis, and neck-to-depth ratio.

ABBREVIATIONS: $\mathrm{ARIC}=$ Atherosclerosis Risk in Communities; $\mathrm{CE}=$ contrast-enhanced; $\mathrm{CFD}=$ computational fluid dynamics; $\mathrm{Cl}=$ confidence interval; $\mathrm{FD}=$ flow divider; IMT = intima-media thickness; $\mathrm{IQ}=$ image quality; MIP = maximum intensity projection; $\mathrm{OR}=$ odds ratio; $\mathrm{TOF}=$ time-of-flight

$\mathbf{T}$ he risk of stroke in patients with carotid atherosclerotic plaque is influenced not only by plaque size and the degree of obstruction but also by the presence of ulceration. ${ }^{1-3}$ Thrombus can form within an ulcer crater as a result of disturbances of nor-

Received October 24, 2011; accepted after revision January 22, 2012.

From the Russell H. Morgan Department of Radiology and Radiological Sciences (M.E., S.K.G., Y.Q., W.M.A.A., B.A.W.), The Johns Hopkins Hospital, Baltimore, Maryland; Department of Mechanical \& Industrial Engineering (Y.H., D.A.S.), University of Toronto, Toronto, Ontario, Canada; Department of Radiology (A.E.N.), University of California Davis Medical Center, Sacramento, California; Departments of Medicine and Population Health Sciences (B.C.A.), University of Wisconsin School of Medicine and Public Health, Madison, Wisconsin; School of Medicine (A.P.), University of Rochester Medical Center, Rochester, New York.

The Atherosclerosis Risk in Communities Study is carried out as a collaborative study supported by National Heart, Lung, and Blood Institute contracts (HHSN268201100005C, HHSN268201100006C, HHSN268201100007C, HHSN268201100008C, HHSN268201100009C, HHSN268201100010C, HHSN268201100011C, and HHSN268201100012C) with the ARIC carotid MRI examination funded by U01HL075572-01. Two of the authors received the support of a Heart \& Stroke Foundation Career Investigator Award (D.A.S.) and Research Fellowship (Y.H.), respectively. mal flow patterns and can embolize to the brain, leading to an ischemic event. ${ }^{1,4}$

Although catheter-based angiography historically has been considered the reference standard for carotid artery assessment, it is limited for identifying ulcers. ${ }^{5-7}$ Noninvasive imaging modalities now offer stenosis measurements with comparable accura$\mathrm{cy}^{8-11}$ and improved ulcer detection. ${ }^{8}$ CTA provides superior spatial resolution to that achievable by MRA, but the exposure to ionizing radiation and the reduction in diagnostic accuracy due to wall calcification ${ }^{12}$ has reinforced the appeal of MRA for carotid disease. This appeal has been further supported by the improved

Some of the results of this study previously presented at: 48th Annual Meeting of the American Society of Neuroradiology, May 17, 2010; Boston, Massachusetts.

Please address correspondence to Bruce A. Wasserman, M.D., Johns Hopkins Hospital, 600 North Wolfe St, 367 East Park Building, Baltimore, MD 21287; e-mail: bwasser@jhmi.edu

- Indicates open access to non-subscribers at www.ajnr.org

$\square$ Indicates article with supplemental on-line videos.

http://dx.doi.org/10.3174/ajnr.A3132 
accuracy of MRA over CTA and sonography compared with DSA in recent meta-analyses. ${ }^{13,14}$

Three-dimensional TOF and CE sequences have become standard MRA techniques used for evaluating carotid stenosis, each with specific advantages. TOF-MRA closely approximates catheter-based stenosis measurements ${ }^{15-17}$ and has the added advantage of not requiring contrast-agent administration, reducing the chance of nephrogenic systemic fibrosis in patients with renal insufficiency. Moreover, TOF-MRA is usually acquired through the carotid bifurcation only and with a higher spatial resolution, as CE-MRA is typically optimized for coverage from the aortic arch to the skull base, and this may enhance the ability of TOFMRA to detect small ulcers. Despite these merits, TOF-MRA is often inadequate for detecting tandem lesions ${ }^{15,16,18}$ and is prone to saturation of slowly flowing or recirculating blood protons, resulting in signal loss within a severely compromised lumen or the carotid bulb, respectively. ${ }^{19,20}$ Recirculating blood proton saturation could also affect the signal within an ulcer crater.

The optimal MRA technique for detecting carotid plaque ulceration has not been established, and its determination was the primary aim of this study. Our focus was on ulcers that can be angiographically detected and our goal was to determine which technique (TOF-MRA or CE-MRA) misses fewer of these ulcers. Because the signal of an ulcer might depend on the hemodynamic pattern of blood flow within the crater (ie, influencing blood proton saturation on TOF-MRA or the mixing with contrast on CEMRA), factors that might influence these flow patterns, such as ulcer orientation, location, and size, were explored.

\section{MATERIALS AND METHODS \\ Subjects}

The ARIC Carotid MR Imaging Study enrolled 2066 participants from 4 communities (Forsyth County, North Carolina; Jackson, Mississippi; suburban Minneapolis, Minnesota; and Washington County, Maryland) to investigate carotid atherosclerotic plaque in a community-representative study including both black and white men and women between the ages of 65 and 84 years in 2005 and 2006. These participants were selected from the larger ARIC cohort, originally recruited in 1987, based on carotid IMT measurements from an earlier sonography examination. Recruitment was designed to include approximately 1200 participants with the highest IMTs (at least $\geq 68$ th percentile at each of 4 sites) and around 800 participants randomly selected from the remaining cohort ( $<68$ th percentile). Details of this study design were previously described. ${ }^{21}$ Institutional review boards of the 4 sites approved this Health Insurance Portability and Accountability Actcompliant study, and all participants gave written informed consent. Of 2066 cases, 600 cases with the thickest plaques, based on maximum wall thickness values acquired on black-blood MR images, as previously described, ${ }^{21}$ were selected for ulcer detection in our study (361 [60\%] men; 505 [84\%] white; mean age, 72 years).

\section{MR Imaging Examination}

A standard imaging protocol was implemented on all participants, and all scans were performed on 1.5T scanners (Minneapolis-Symphony Maestro, Siemens, Erlangen, Germany; 3 other
sites-Excite platform, GE Medical Systems, Milwaukee, Wisconsin) using bilateral 4-element phased array carotid coils (Machnet, the Netherlands). ${ }^{21}$

A 3D TOF-MRA was acquired through both carotid bifurcations in the axial plane with the following parameters: TR/TE/flip angle, $25 \mathrm{~ms} / 3.3-4.7 \mathrm{~ms} / 25^{\circ}$; 2 signal averages; acquired matrix, $256 \times 256$ zero-padded to $512 \times 512$; FOV, $150 \mathrm{~mm} \times 150 \mathrm{~mm}$; section thickness, $2 \mathrm{~mm}$ without overlap; acquisition time, $4-5$ minutes.

Two-dimensional black-blood MR images were acquired through the long axes of both carotid bifurcations and used as a scout to orient a stack of 16 parallel axial 2D black-blood MR images through the carotid artery with the thicker wall or plaque (2-mm section thickness, section gap: $0 \mathrm{~mm} ; 3.2 \mathrm{~cm}$ coverage; acquired resolution $0.51 \times 0.58 \times 2 \mathrm{~mm}^{3}$ ).

A $3 \mathrm{D}$ CE-MRA was acquired in the coronal plane after the intravenous injection of $0.1 \mathrm{mmol} / \mathrm{kg}$ gadodiamide (Omniscan, GE Healthcare) by power injector with the following parameters at the 3 sites with GE scanner: TR/TE/flip angle, $5.9 \mathrm{~ms} / 1.7 \mathrm{~ms} / 29^{\circ}$; 0.5 signal average; section thickness, $2 \mathrm{~mm}$ with $1-\mathrm{mm}$ overlap; FOV , $200 \mathrm{~mm} \times 200 \mathrm{~mm}$; and acquired matrix, $256 \times 160$, zeropadded to $512 \times 512$. The protocol at the sites with Siemens scanner was the same, except for section thickness, $1 \mathrm{~mm}$ without overlap; 1 signal average; FOV, $160 \mathrm{~mm} \times 120 \mathrm{~mm}$; and acquired matrix, $320 \times 168$, zero-padded to $640 \times 480$. At the Siemens scanner site, the CE-MRA acquisition was timed by bolus tracking, whereas at the GE scanner sites, 3 acquisitions (phases) were acquired, with the first acquired 10 seconds after the start of contrast administration. The phase that demonstrated the best arterial contrast was used for analysis. Acquisition time was approximately 30 seconds for each phase. The axial 2D black-blood MR images were repeated beginning 5 minutes after contrast administration. MIP images were generated for both CE-MRA and TOF-MRA sequences.

\section{Image Analysis}

Two experienced analysts (A.P. and M.E.) were trained by a senior neuroradiologist to interpret each MR imaging series and identify ulcers. Analysts were certified based on correctly interpreting a series of challenging test cases. They independently determined ulcer presence by evaluating all available MR images, including TOF-MRA and CE-MRA source images and MIP images, as well as long-axis and pre- and postcontrast short-axis high-resolution black-blood MR images for all 600 cases on the side selected for black-blood MR imaging (ie, the thicker wall/plaque). An ulcer was defined as a niche in the plaque surface measuring $\geq 2 \mathrm{~mm}$ in depth. ${ }^{22-24}$ CE-MRA and TOF-MRA images were evaluated using short- and long-axis reconstructions of each 3D MRA dataset, along with the MIP images. The black-blood MR images, acquired to achieve a comprehensive characterization of the vessel wall, were used here to identify ulcers in conjunction with the MRA findings. ${ }^{25}$ These black-blood MR images also ensured that ulcerlike contour irregularities of the carotid artery that actually represented a normal arterial wall with adjacent isolated plaque $^{6,26}$ were not defined as ulcers. A senior neuroradiologist (B.A.W.) arbitrated any disagreements and a consensus was reached for each case. IQ scores for CE- and TOF-MRA 


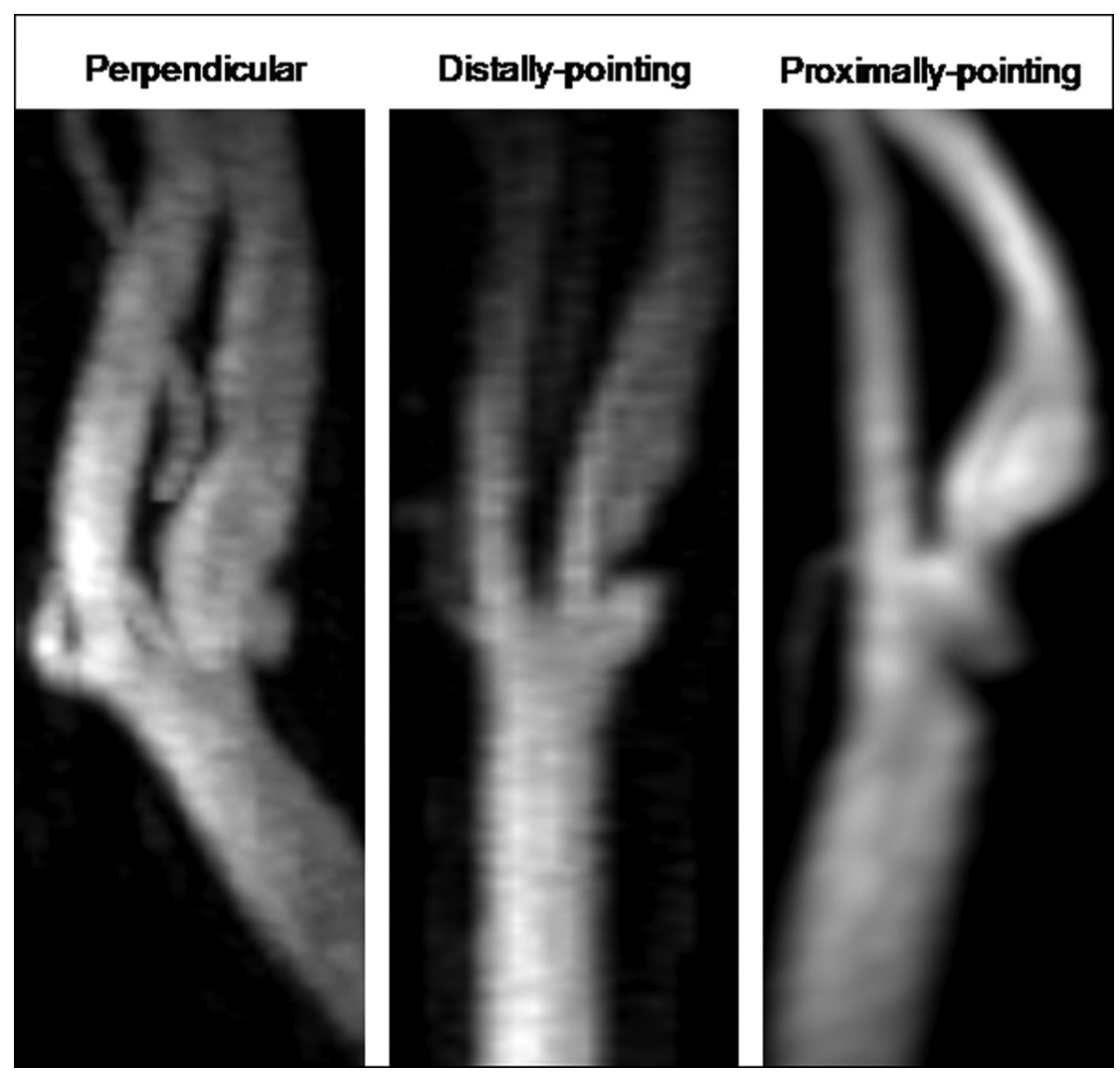

FIG 1. Examples of 3 ulcer orientations shown on CE-MRA MIP images. Perpendicular: If a line is to be drawn connecting the center of the ulcer opening and its tip, it will be perpendicular to the vessel axis. Distally pointing: Ulcer tip is distal to its opening. Proximally pointing: Ulcer tip is proximal to its opening.
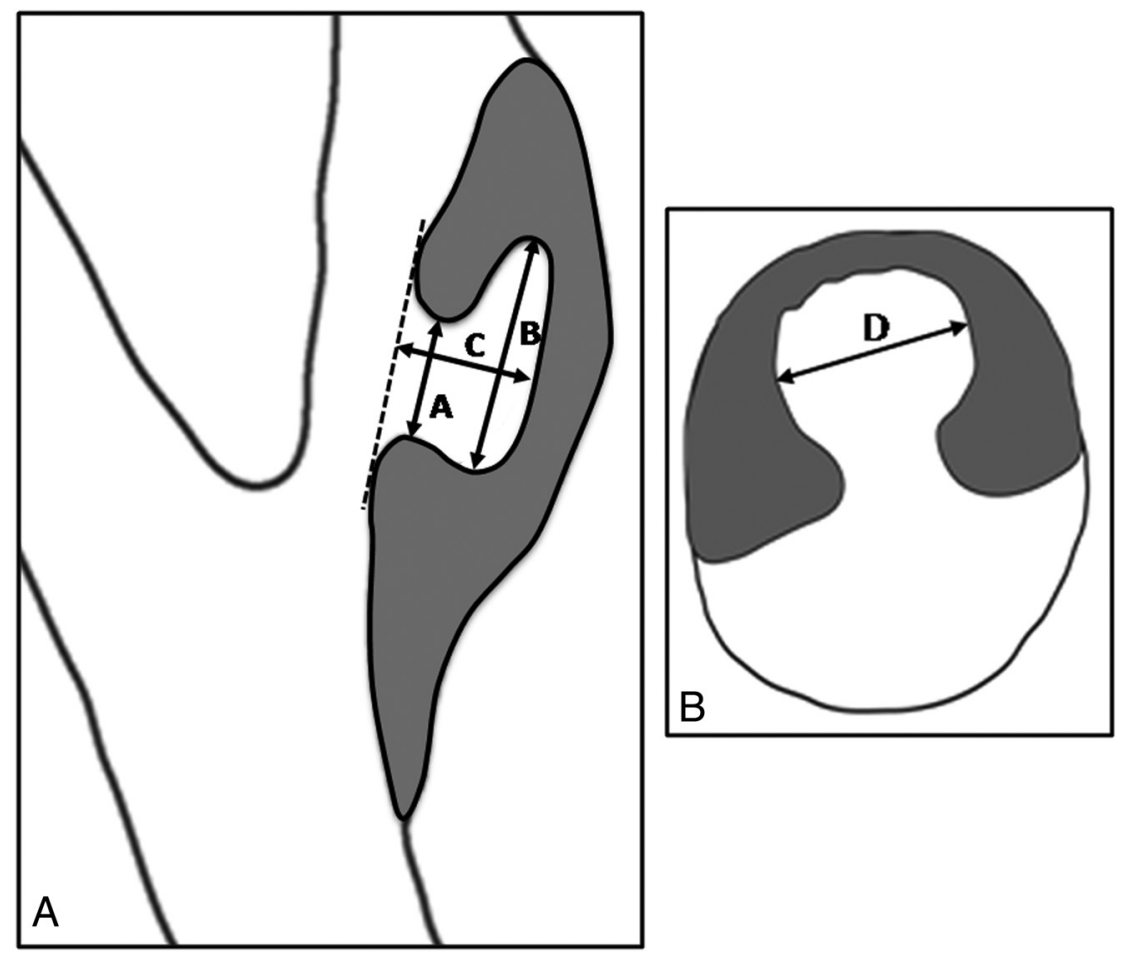

FIG 2. Technique for measuring ulcer dimensions. Ulcer neck (A), maximum craniocaudal dimension (B) and depth (C) were measured on CE-MRA MIP images based on the view that shows the largest dimensions (Fig 2A). Maximum transverse dimension (D) was measured on the reconstructed CE-MRA source image oriented perpendicular to the vessel axis that shows the largest transverse dimension (Fig 2B). images were assigned using a scale from 0 to 2 ( 0 , poor artery visualization with artifact and/or noise resulting in a noninterpretable image; 1 , adequate arterial visualization with artifact and/or noise that does not interfere with interpretation; 2, good arterial visualization with little to no artifact and/or noise resulting in a confident interpretation). Ulcer orientation was categorized into 3 groups: perpendicular, distally pointing, and proximally pointing (Fig 1). In addition, we categorized ulcer location as proximal, isolevel, or distal to the point of maximum stenosis and to the FD. The circumferential location of the ulcer was also recorded (ie, along FD, opposite FD, or on sidewalls). Ulcer cavity and neck dimensions were measured on CE-MRA MIP and source images (Fig 2). An index of ulcer volume was calculated based on the following equation:

\section{Ulcer volume}

$=$ maximum craniocaudal dimension

$\times$ maximum transverse dimension

$$
\times \text { depth }
$$

Carotid stenosis was measured based on NASCET guidelines. ${ }^{27}$

Sixty cases of ulcerated carotid arteries were identified. These 60 ulcerated arteries, along with 40 nonulcerated arteries randomly sampled from the remaining 540 cases, were assigned to 2 neuroradiologists (S.K.G. and A.E.N.) for evaluation of ulcer presence. All images were de-identified and assigned in random order. The readers were blinded to the study objectives and evaluated only 1 sequence (CE-MRA or TOF-MRA) at a time without access to other sequences or knowledge of clinical information. MIP and source images were reviewed together for each case and the readers were allowed to generate multiplanar reconstructions of the source images. The readers were told which side to analyze (ie, the thicker side selected for black-blood MRI). CE-MRA and TOF-MRA series for the same case were interpreted in separate sessions, with at least 2 weeks between evaluations to prevent reader recall. Each reader examined all 100 cases and recorded the location of detected ulcers (ie, distance from FD and the position on the axial plane based on a clockwise model). An investigator (M.E.) subsequently matched corresponding ulcers detected by each reader on the CE- and TOF-MRA series using their recorded 
locations. Images were analyzed using a PACS workstation (Ultravisual, Emageon, Alabama).

\section{Statistical Analysis}

A paired $t$ test was used to compare IQ scores between CE- and TOF-MRAs for each case. Percents of ulcers detected by CE- and TOF-MRA techniques were calculated separately for each reader. Intraobserver agreement of the number of ulcers detected for each artery was estimated based on 20 repeat readings for each reader, separated by at least 3 weeks to prevent reader recall, and interobserver agreement was estimated based on all 100 cases. Agreement for CE and TOF-MRA results was estimated separately using weighted $\kappa$ coefficients. A $\kappa$ value of $<0.4$ was characterized as poor agreement; $0.4-0.75$ as fair to good; and $>0.75$ as excellent. ${ }^{28}$ Logistic regression was used to estimate correlation of TOF-MRA ulcer detection with ulcer orientation, location, volume, neck-to-depth ratio, as well as IQ score and degree of stenosis. We applied robust variance estimates to account for 2 readers' results for each ulcer. Analysis was completed using SPSS 17.0 (SPSS, Chicago, Illinois) and STATA 10.0 (Stata, College Station, Texas).

\section{Computational Fluid Dynamics}

To illustrate the effect of ulcer orientation on blood flow patterns within the ulcer, we performed CFD simulations of pulsatile flow in an idealized carotid bifurcation with a $30 \%$ eccentric stenosis, as previously described, ${ }^{29}$ to which we attached representative distally and proximally pointing ellipsoidal ulcers. ${ }^{30}$ Flow patterns were visualized by randomly seeding 250,000 tracer particles within and away from the ulcer and tracking their evolution over 3 cardiac cycles, using previously reported techniques. ${ }^{31}$

\section{RESULTS}

\section{Study Group Ulcerations}

Sixty ulcerated carotid arteries were identified when evaluating all available MR images. MRA images in 4 cases (3 TOF-MRAs; 1 CE-MRA) were noninterpretable (ie, IQ score $=0$ ) due to motion and were excluded, leaving 56 arteries for analysis. Average IQ score was higher for CE-MRA than TOF-MRA (mean $\pm \mathrm{SD}$ : $1.83 \pm 0.40$ versus $1.72 \pm 0.51, P=.03)$. Some plaques had multiple ulcers ( 7 arteries had 2 ulcers per artery, and 4 arteries had 3 ulcers per artery) for a total of 71 ulcers.

\section{Reliability}

Interobserver agreement for ulcer detection was excellent $(\kappa=$ 0.86 [95\% CI, 0.77-0.95]) for CE-MRA, and good $(\kappa=0.72$ [95\% CI, $0.58-0.86])$ for TOF-MRA. Average intraobserver reliability for the 2 readers for both CE- and TOF-MRAs was excellent $(\kappa=$ 0.91 [95\% CI $0.78-1.00$ ], and $\kappa=0.85$ [95\% CI, 0.69-1.00], respectively).

\section{Comparison of Ulcer Detection by CE-MRA and TOF-MRA}

Each reader detected more ulcers on CE-MRA than TOF-MRA. Table 1 presents ulcer detection results for each MRA technique for individual ulcers and vessels for the 2 readers. As an average, of 71 ulcers, readers detected $55 \%$ on both TOF-MRA and CE-MRA, $37.5 \%$ only on CE-MRA, and $1.5 \%$ only on TOF-MRA, missing $6 \%$ of ulcers by both methods. No ulcers were detected on TOF-
Table 1: Ulcer detection by CE-MRA and TOF-MRA techniques for individual ulcers and vessels for 2 readers

\begin{tabular}{lcc}
\hline & $\begin{array}{c}\text { Reader } \mathbf{1} \\
\text { Number (\%) }\end{array}$ & $\begin{array}{c}\text { Reader } 2 \\
\text { Number (\%) }\end{array}$ \\
\hline Individual ulcer detection & & \\
Both CE and TOF & $45(63)$ & $33(46.5)$ \\
Only CE & $24(34)$ & $29(41)$ \\
Only TOF & $1(1.5)$ & $1(1.5)$ \\
Neither CE nor TOF & $1(1.5)$ & $8(11)$ \\
Total (all MR sequences) & $71(100)$ & $71(100)$ \\
Vessels detected as ulcerated & & \\
Both CE and TOF & $41(73)$ & $30(54)$ \\
Only CE & $14(25)$ & $23(41)$ \\
Only TOF & $1(2)$ & $1(2)$ \\
Neither CE nor TOF & $0(0)$ & $2(3)$ \\
Total (all MR sequences) & $56(100)$ & $56(100)$ \\
\hline
\end{tabular}

MRA in an average of $33 \%$ of vessels with ulcerated plaques detected on CE-MRA. For the 1 ulcer missed on CE-MRA but detected on TOF-MRA by both readers, the CE-MRA IQ score was 1 , because of motion and low arterial contrast, whereas the TOFMRA IQ was 2, and a carotid body tumor was incidentally noted. Although the tumor did not obscure the ulcer, it was noted to be a distraction. For 11 vessels with multiple ulcers, no ulcer was detected on TOF-MRA in 4 and 5 vessels by reader 1 and reader 2 , respectively, whereas all ulcers in these vessels were detected on CE-MRA by both readers. Readers 1 and 2 identified 2 and 1 cases, respectively, of an ulcer-like configuration on the CE-MRA sequence, caused by a plaque indenting the base of the bulb, without evidence for a true ulcerated plaque as confirmed by the blackblood MR imaging. These cases were not identified as ulcerated Table 2: Characteristics of 69 ulcers detected by CE-MRA by
reader 1 and TOF-MRA ulcer-detection percent for each characteristic

\begin{tabular}{|c|c|c|}
\hline Characteristics & $\begin{array}{l}\text { Detected by } \\
\text { CE-MRA }^{\mathrm{a}}\end{array}$ & $\begin{array}{l}\text { Detected by } \\
\text { TOF-MRA }{ }^{\text {b }} \text { (among } \\
\text { Those Detected } \\
\text { by CE-MRA) }\end{array}$ \\
\hline \multicolumn{3}{|l|}{ IQ score } \\
\hline 1 & $28(41)$ & $18(64)$ \\
\hline 2 & $41(59)$ & $27(66)$ \\
\hline \multicolumn{3}{|l|}{ Ulcer orientation ${ }^{c}$} \\
\hline Perpendicular & $47(68)$ & $28(60)$ \\
\hline Distally pointing & $16(23)$ & $15(94)$ \\
\hline Proximally pointing & $6(9)$ & $2(33)$ \\
\hline \multicolumn{3}{|l|}{$\begin{array}{l}\text { Ulcer location relative to point } \\
\text { of maximum stenosis }\end{array}$} \\
\hline Proximal & $36(52)$ & $26(72)$ \\
\hline Isolevel & $14(20)$ & $5(36)$ \\
\hline Distal & $19(28)$ & $14(74)$ \\
\hline \multicolumn{3}{|l|}{ Ulcer location relative to FD } \\
\hline Proximal & $18(26)$ & $13(72)$ \\
\hline Isolevel & $28(41)$ & $19(68)$ \\
\hline Distal & $23(33)$ & $13(57)$ \\
\hline \multicolumn{3}{|l|}{ Ulcer circumferential location } \\
\hline Opposite FD & $44(64)$ & $29(66)$ \\
\hline Along FD & $0(0)$ & $0(\mathrm{~N} / \mathrm{A})$ \\
\hline On sidewalls & $25(36)$ & $16(64)$ \\
\hline Ulcer neck-to-depth ratio ${ }^{c}$ & $1.61(0.56)$ & $1.71(0.57)$ \\
\hline Ulcer volume index $\left(\mathrm{mm}^{3}\right)^{c}$ & $67.63(66.50)$ & $81.83(75.09)$ \\
\hline Degree of stenosis $(\%)^{c}$ & $27.16(18.06)$ & $23.4(16.10)$ \\
\hline
\end{tabular}

a Data are presented as number of ulcers (\% of 69 ) or mean (SD).

${ }^{b}$ Data are presented as number of ulcers (\% in category) or mean (SD).

${ }^{c} P<.05$ in univariable regression model. 
Table 3: Multivariable logistic regression model for ulcer detection by TOF-MRA among ulcers detected by CE-MRA

\begin{tabular}{|c|c|c|c|}
\hline \multirow[b]{2}{*}{ Characteristic } & \multicolumn{3}{|c|}{ Multivariable } \\
\hline & OR & $95 \% \mathrm{Cl}$ & $P$ \\
\hline $\begin{array}{l}\text { Orientation (distally pointing vs } \\
\text { perpendicular) }\end{array}$ & 5.57 & $1.08-28.65$ & 0.04 \\
\hline $\begin{array}{l}\text { Orientation (proximally-pointing vs } \\
\text { perpendicular) }\end{array}$ & 0.21 & $0.14-0.29$ & $<0.001$ \\
\hline Location (proximal vs isolevel) ${ }^{a}$ & 2.79 & $0.94-8.32$ & 0.06 \\
\hline Location (distal vs isolevel) ${ }^{a}$ & 5.17 & $2.10-12.70$ & $<0.001$ \\
\hline Neck-to-depth ratio & 1.96 & $1.11-3.45$ & 0.02 \\
\hline Volume index & 1.01 & $1.00-1.02$ & 0.06 \\
\hline Degree of stenosis & 0.97 & $0.94-1.01$ & 0.23 \\
\hline
\end{tabular}

Note:- Robust variance estimate was used to account for 2 readers' results for each ulcer.

a Ulcer location relative to point of maximum stenosis.

on the TOF-MRA sequence. For the purpose of comparing TOFand CE-MRA techniques, these arteries were considered nonulcerated.

\section{Associations between Ulcer Characteristics and its Detection by TOF-MRA}

Because only 1 ulcer detected by TOF-MRA was missed on CEMRA, we restricted our assessment to factors influencing TOFMRA ulcer detection among ulcers detected by CE-MRA. Table 2 shows the characteristics of 69 ulcers detected by CE-MRA and percents of ulcer detection by TOF-MRA for each characteristic for reader 1 . Most ulcers (68\%) were perpendicular to the vessel axis, located proximal to the point of maximum stenosis (52\%) and on the opposite FD wall (64\%). There were no mushroom- shaped ulcers. The 1 ulcer missed on CE-MRA but detected on TOF-MRA was perpendicular to the vessel axis, located isolevel to the point of maximum stenosis and on the opposite FD wall.

Univariate logistic regression demonstrated significant associations between ulcer detection by TOF-MRA and ulcer orientation, location relative to the point of maximum stenosis, neck-todepth ratio, volume, and degree of stenosis, but not with IQ score, ulcer location relative to FD, and circumferential location. A multivariate logistic regression model for ulcer detection by TOFMRA, adjusting for all characteristics found to be associated with ulcer detection by the univariate models confirmed the association of ulcer orientation, location relative to the point of maximum stenosis, and neck-to-depth ratio (Table 3 ).

Ulcer orientation had a strong association with ulcer detection by TOF-MRA. Distally pointing ulcers were positively associated with ulcer detection by TOF-MRA (OR $=5.57,95 \% \mathrm{CI}=1.08-$ 28.65; Fig $3 A$ ), whereas proximally pointing ulcers were negatively associated $(\mathrm{OR}=0.21,95 \% \mathrm{CI}=0.14-0.29$; Fig $3 B)$. Ulcers isolevel to the point of maximum stenosis were detected less frequently by TOF-MRA than ulcers proximal or distal to the point of maximum stenosis. Ulcers with a higher neck-to-depth ratio were detected more frequently by TOF-MRA.

\section{Computational Fluid Dynamics}

As Fig 4 and On-Line Videos 1 and 2 show, most particles leave the ulcer by the end of 1 cardiac cycle; however, some remain and circulate slowly within the ulcer tip. For the distally pointing ulcer, high velocities fill nearly the entire ulcer during systole,

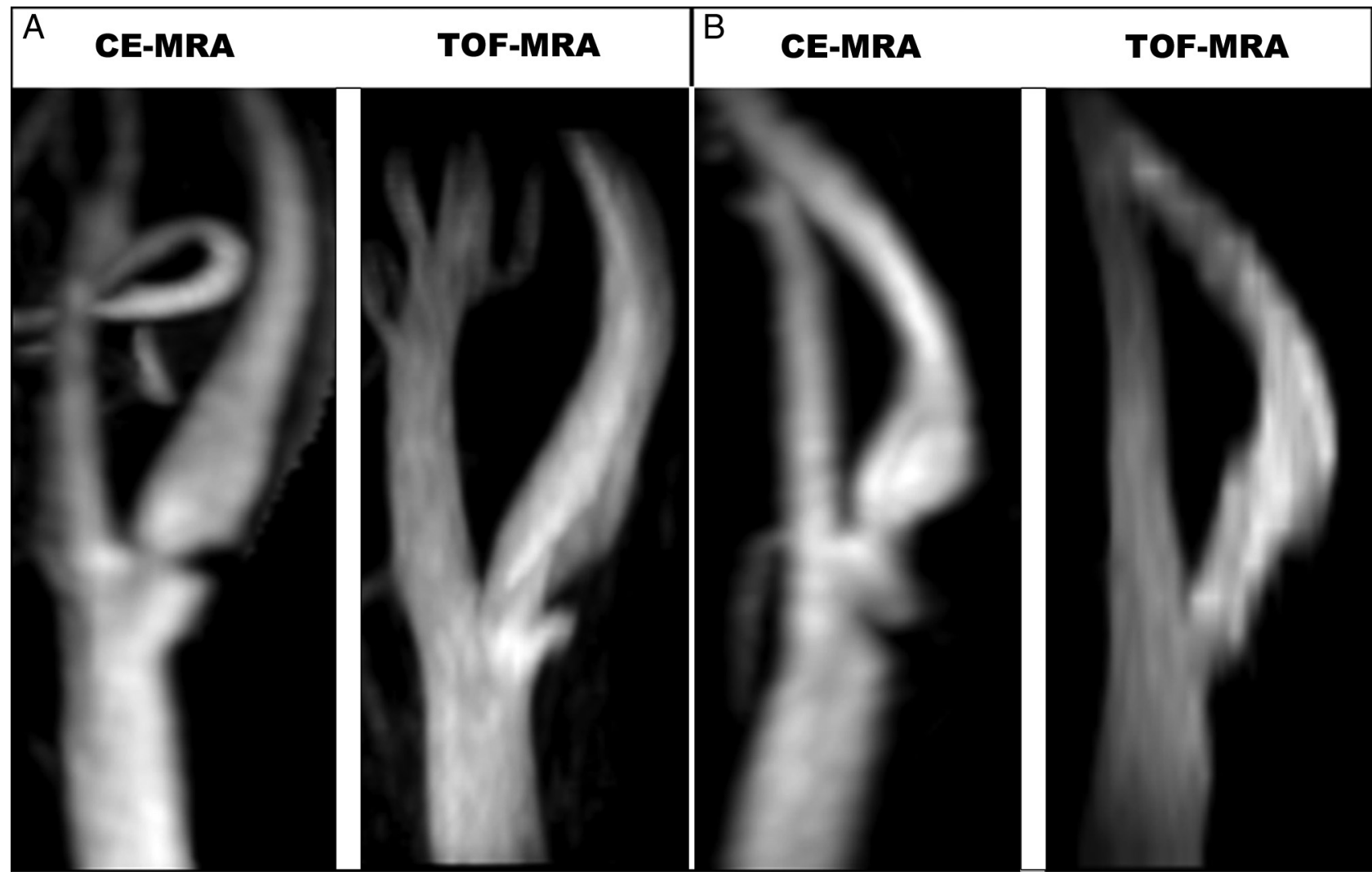

FIG 3. MIP images of a distally pointing ulcer $(A)$ detected on both CE-MRA and TOF-MRA, and a proximally pointing ulcer $(B)$ detected on CE-MRA but not on TOF-MRA. 

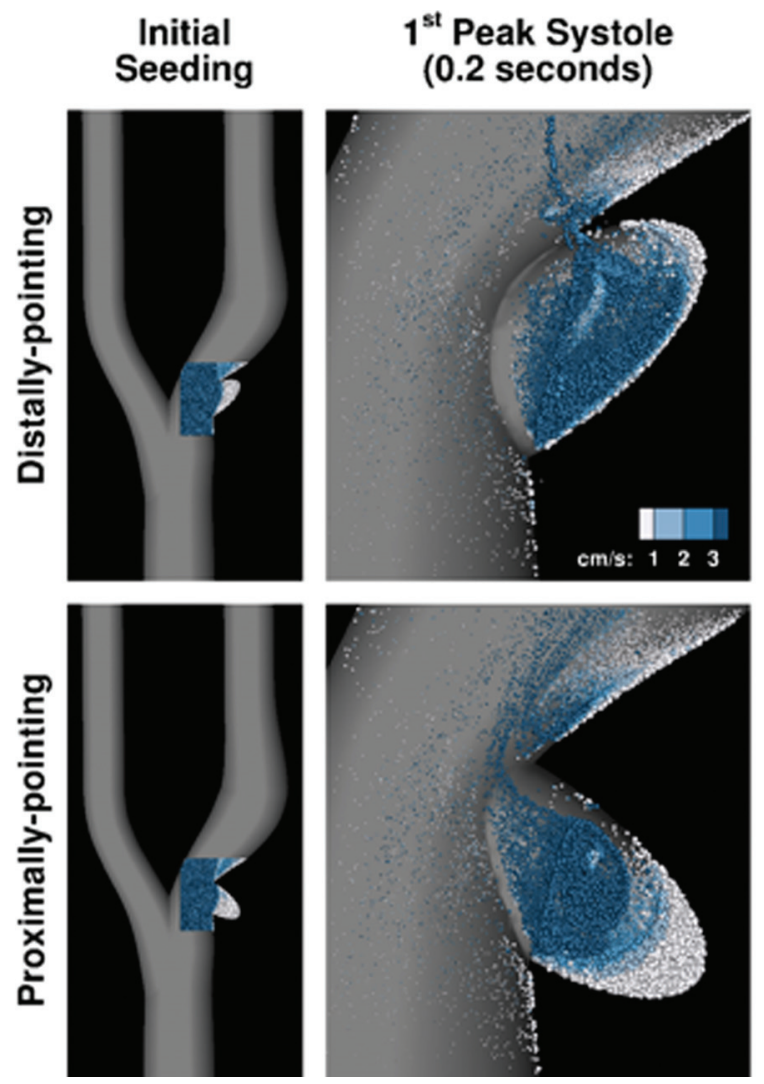

$2^{\text {nd }}$ Peak Systole

(1.2 seconds)
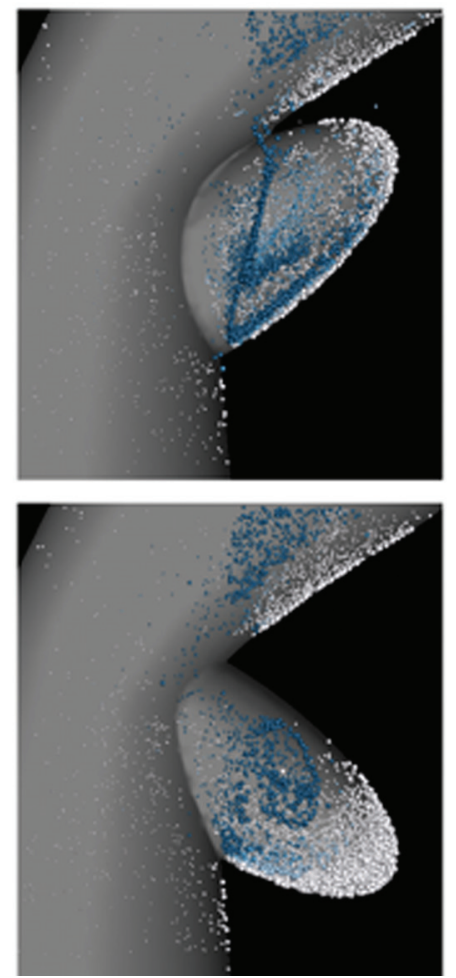

$3^{\text {rd }}$ Peak Systole (2.2 seconds)
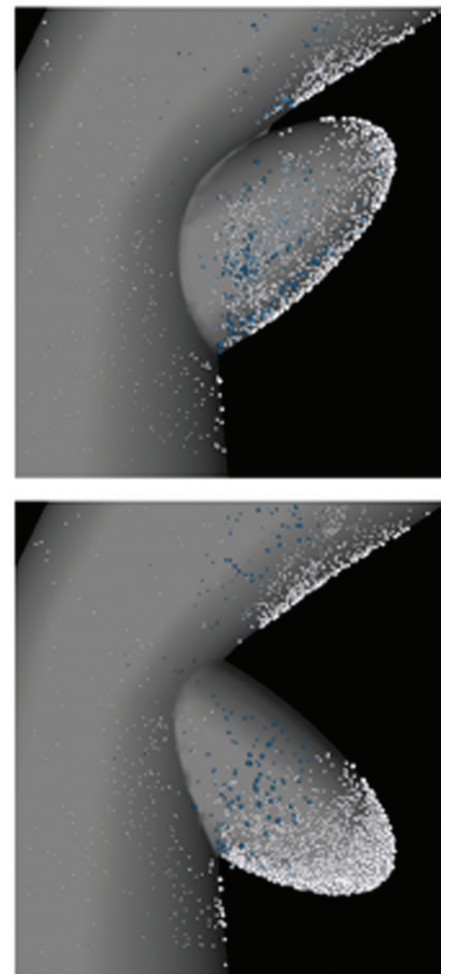

FIG 4. Visualization of flow patterns computed in a $30 \%$-stenosed carotid bifurcation with distally and proximally pointing ellipsoidal ulcers. The leftmost column shows the full CFD models and the initial distribution of particles seeded at end diastole. Subsequent columns show the distribution of particles remaining after each peak systole, with the amount of time since seeding (ie, residence time) also indicated. Colors are used to indicate particle velocity in $\mathrm{cm} / \mathrm{s}$, and size indicates the depth of the particles such that large particles are those closest to the symmetry plane and small particle are those closest to the walls.

whereas for the proximally pointing ulcer, high velocities are more confined to the ulcer opening. The net effect is a larger volume of long-residing particles at the ulcer tip for the latter case.

\section{DISCUSSION}

Our results indicate that CE-MRA detects more carotid plaque ulcers than TOF-MRA. Although TOF-MRA can adequately measure carotid stenosis, it detected no ulcers in approximately one-third of ulcerated carotid plaques detected on CE-MRA, even when multiple ulcers were present in a plaque. This highlights the importance of CE-MRA for carotid plaque evaluation, because ulceration is a predictor of impending stroke even after adjusting for stenosis. ${ }^{2,3}$

To our knowledge, our study is the first population-based study of geometric ulcer characteristics. Our observations extended those previously reported, namely, that perpendicular followed by distally pointing orientations were encountered most frequently, along with a location proximal to the point of maximum stenosis. ${ }^{32-35}$ We did not encounter mushroom-shaped ulcers, possibly because our study population did not select for advanced disease, as was done in the prior reports. ${ }^{32-35}$

Our study shows that ulcer detection by TOF-MRA depends on ulcer orientation, location, and neck-to-depth ratio. The influence of these ulcer characteristics on ulcer detection by TOFMRA can be explained by differences in hemodynamic patterns within the ulcer cavity that affect spin saturation. The general pattern of blood flow in ulcers has been described by Imbesi and Kerber $^{4}$ for a carotid artery with a perpendicular ulcer. The authors showed that the blood stream partly enters the distal end of the ulcer opening, swirls inside the ulcer pouch opposing the direction of the blood stream in the parent artery, and partly leaves the ulcer, much like flow in the classic driven cavity problem in fluid mechanics. ${ }^{36}$ In this context, our observation that the neckto-depth ratio affects ulcer detection on TOF-MRA is consistent with the fact that narrow, deeper cavities tend to harbor larger and slower regions of recirculating flow subject to spin saturation; and also that missed ulcers tend to be isolevel to the stenosis, that is, when the prevailing flow tends to be directed perpendicular to the ulcer opening, as for a driven cavity flow. The fact that our CFD simulations predict a larger volume of longer residing particles for the proximally pointing ulcer case is consistent with the observation that TOF-MRA is more prone to signal loss when the ulcer is pointing away from the blood stream in the parent artery. As suggested by Imbesi and Kerber, ${ }^{4}$ long-residing platelets in the ulcer cavity more likely aggregate and form thrombus, which can be sucked into the bloodstream based on the Bernoulli effect. Thus, ulcers detected on CE-MRA but missed on TOF-MRA may be at greater risk for clinical events related to thrombus formation.

The limitations of our study include the following: First, histologic validation of ulcer presence was not possible and some 
ulcers may have been missed, even on CE-MRA imaging. This is particularly true for shallow, intimal ulcerations and for tiny ulcers embedded in plaque surface irregularities. ${ }^{6}$ This is a consequence of the main objective of this study, which is to analyze ulcers that are angiographically detectable. We could not effectively compare TOF- and CE-MRA techniques using ulcers that can only be detected by specimen analysis. These MRA techniques are also potentially subject to falsely identifying ulcers, as when a segment of normal vessel wall exists between 2 plaques. ${ }^{6,26} \mathrm{We}$ combined high-resolution black-blood MR imaging and MRA techniques (including MIP and source images) to maximize our ability to detect all ulcers ${ }^{25}$ and to ensure that these ulcer-like appearances were not counted as true ulcers. Furthermore, the emphasis of our study was a comparison of techniques, and we did not attempt to estimate the positive predictive value of either MRA method.

Second, the lower IQ scores observed for TOF-MRA might have interfered with ulcer detection. The lower scores may have been the consequence of the longer acquisition time for TOFMRA, making it more prone to motion-related artifact compared with the CE-MRA technique. After excluding the noninterpretable cases (IQ score $=0$ ), there was no association between IQ score and ulcer detection by TOF-MRA. Nevertheless, we noticed lower IQ score related to motion artifacts was a possible reason for some missed ulcers on TOF-MRA, rather than the hemodynamic basis described previously.

\section{CONCLUSIONS}

TOF-MRA misses as many as one-third of carotid plaque ulcers that can be detected by CE-MRA, and these misses are influenced by hemodynamic patterns of blood flow that depend on ulcer orientation (proximally pointing), position relative to narrowing, and geometry (low neck-to-depth ratio). These patterns of flow that lead to saturation of proton spins and signal loss on TOFMRA might also create an environment conducive to thrombus formation and influence its risk for stroke, though further work is needed to establish an association with this potential consequence.

\section{ACKNOWLEDGMENTS}

Two of the authors acknowledge the support of a Heart \& Stroke Foundation Career Investigator Award (D.A.S.) and Research Fellowship (Y.H.), respectively. The authors thank Emily Wong for guidance in the design of the CFD ulcer models. The authors also thank the staff and participants of the ARIC study for their important contributions.

\section{REFERENCES}

1. Hennerici MG. The unstable plaque. Cerebrovasc Dis 2004;17:17-22

2. Rothwell PM, Gibson R, Warlow CP. Interrelation between plaque surface morphology and degree of stenosis on carotid angiograms and the risk of ischemic stroke in patients with symptomatic carotid stenosis. On behalf of the European Carotid Surgery Trialists' Collaborative Group. Stroke 2000;31:615-21

3. Eliasziw M, Streifler JY, Fox AJ, et al. Significance of plaque ulceration in symptomatic patients with high-grade carotid stenosis. North American Symptomatic Carotid Endarterectomy Trial. Stroke 1994;25:304-08

4. Imbesi SG, Kerber CW. Why do ulcerated atherosclerotic carotid artery plaques embolize? A flow dynamics study. AJNR Am J Neuroradiol 1998;19:761-66

5. Streifler JY, Eliasziw M, Fox AJ, et al. Angiographic detection of carotid plaque ulceration. Comparison with surgical observations in a multicenter study. North American Symptomatic Carotid Endarterectomy Trial. Stroke 1994;25:1130-32

6. Edwards JH, Kricheff II, Riles T, et al. Angiographically undetected ulceration of the carotid bifurcation as a cause of embolic stroke. Radiology 1979;132:369-73

7. Eikelboom BC, Riles TR, Mintzer R, et al. Inaccuracy of angiography in the diagnosis of carotid ulceration. Stroke 1983;14:882-85

8. Randoux B, Marro B, Koskas F, et al. Carotid artery stenosis: prospective comparison of $\mathrm{CT}$, three-dimensional gadolinium-enhanced MR, and conventional angiography. Radiology 2001;220: $179-85$

9. Nederkoorn PJ, van der Graaf Y, Hunink MG. Duplex ultrasound and magnetic resonance angiography compared with digital subtraction angiography in carotid artery stenosis: a systematic review. Stroke 2003;34:1324-32

10. Nederkoorn PJ, Mali WP, Eikelboom BC, et al. Preoperative diagnosis of carotid artery stenosis: accuracy of noninvasive testing. Stroke 2002;33:2003-08

11. U-King-Im JM, Trivedi R, Cross J, et al. Conventional digital subtraction $\mathrm{x}$-ray angiography versus magnetic resonance angiography in the evaluation of carotid disease: patient satisfaction and preferences. Clin Radiol 2004;59:358-63

12. Leclerc X, Godefroy O, Pruvo J, et al. Computed tomographic angiography for the evaluation of carotid artery stenosis. Stroke 1995; 26:1577-81

13. Chappell FM, Wardlaw JM, Young GR, et al. Carotid artery stenosis: accuracy of noninvasive tests individual patient data meta-analysis. Radiology 2009;251:493-502

14. Wardlaw JM, Chappell FM, Best JJ, et al. Non-invasive imaging compared with intra-arterial angiography in the diagnosis of symptomatic carotid stenosis: a meta-analysis. Lancet 2006;367:1503-12

15. Fellner C, Lang W, Janka R, et al. Magnetic resonance angiography of the carotid arteries using three different techniques: accuracy compared with intraarterial $x$-ray angiography and endarterectomy specimens. J Magn Reson Imaging 2005;21:424-31

16. Muhs BE, Gagne P, Wagener J, et al. Gadolinium-enhanced versus time-of-flight magnetic resonance angiography: what is the benefit of contrast enhancement in evaluating carotid stenosis? Ann Vasc Surg 2005; 19:823-28

17. Slosman F, Stolpen AH, Lexa FJ, et al. Extracranial atherosclerotic carotid artery disease: evaluation of non-breath-hold three-dimensional gadolinium-enhanced MR angiography. AJR Am J Roentgenol 1998;170:489-95

18. Wutke R, Lang W, Fellner C, et al. High-resolution, contrast-enhanced magnetic resonance angiography with elliptical centric $k$ space ordering of supra-aortic arteries compared with selective $\mathbf{x}$ ray angiography. Stroke 2002;33:1522-29

19. Nederkoorn PJ, van der Graaf Y, Eikelboom BC, et al. Time-of-flight MR angiography of carotid artery stenosis: does a flow void represent severe stenosis? AJNR Am J Neuroradiol 2002;23:1779-84

20. Ahn KJ, You WJ, Lee JH, et al. Re-circulation artefact at the carotid bulb can be differentiated from true stenosis. Br J Radiol 2004;77: $551-56$

21. Wasserman BA, Astor BC, Sharrett AR, et al. MRI measurements of carotid plaque in the Atherosclerosis Risk in Communities (ARIC) Study: methods, reliability and descriptive statistics. J Magn Reson Imaging 2010;31:406-15

22. Troyer A, Saloner D, Pan XM, et al. Major carotid plaque surface irregularities correlate with neurologic symptoms. J Vasc Surg 2002; 35:741-47

23. Polak JF, O'Leary DH, Kronmal RA, et al. Sonographic evaluation of carotid artery atherosclerosis in the elderly: relationship of disease severity to stroke and transient ischemic attack. Radiology 1993;188: 363-70 
24. Anderson GB, Ashforth R, Steinke DE, et al. CT angiography for the detection and characterization of carotid artery bifurcation disease. Stroke 2000;31:2168-74

25. Yu W, Underhill HR, Ferguson MS, et al. The added value of longitudinal black-blood cardiovascular magnetic resonance angiography in the cross sectional identification of carotid atherosclerotic ulceration. J Cardiovasc Magn Reson 2009;18:31

26. Edwards JH, Kricheff II, Gorstein F, et al. Atherosclerotic subintimal hematoma of the carotid artery. Radiology 1979;133:123-29

27. Beneficial effect of carotid endarterectomy in symptomatic patients with high-grade carotid stenosis. North American Symptomatic Carotid Endarterectomy Trial Collaborators. $N$ Engl J Med 1991;325:445-53

28. Fleiss J. Statistical Methods for Rates and Proportions. 2nd ed. New York: John Wiley \& Sons, 1981; p. 218

29. Steinman DA, Poepping TL, Tambasco M, et al. Flow patterns at the stenosed carotid bifurcation: effect of concentric versus eccentric stenosis. Ann Biomed Eng 2000;28:415-23

30. Wong EY, Nikolov HN, Thorne ML, et al. Clinical Doppler ultrasound for the assessment of plaque ulceration in the stenosed ca- rotid bifurcation by detection of distal turbulence intensity: a matched model study. Eur Radiol 2009;19:2739-49

31. Tambasco M, Steinman DA. Path-dependent hemodynamics of the stenosed carotid bifurcation. Ann Biomed Eng 2003;31:1054-65

32. Lovett JK, Gallagher PJ, Hands LJ, et al. Histological correlates of carotid plaque surface morphology on lumen contrast imaging. Circulation 2004;110:2190-97

33. de Weert TT, Cretier S, Groen HC, et al. Atherosclerotic plaque surface morphology in the carotid bifurcation assessed with multidetector computed tomography angiography. Stroke 2009;40: $1334-40$

34. Lovett JK, Rothwell PM. Site of carotid plaque ulceration in relation to direction of blood flow: an angiographic and pathological study. Cerebrovasc Dis 2003;16:369-75

35. Saba L, Caddeo G, Sanfilippo R, et al. CT and ultrasound in the study of ulcerated carotid plaque compared with surgical results: potentialities and advantages of multidetector row $\mathrm{CT}$ angiography. AJNR Am J Neuroradiol 2007;28:1061-66

36. Shankar PN, Deshpande MD. Fluid mechanics in the driven cavity. Annu Rev Fluid Mech 2000;32:93-136 